\title{
Eugenol como anestésico para jundiá (Rhamdia voulezi) em diferentes pesos
}

\section{Eugenol as anesthetic for silver catfish (Rhamdia voulezi) with different weight}

\author{
Odair Diemer ${ }^{1 *}$; Dacley Hertes Neu²; Fabio Bittencourt"; \\ Altevir Signor ${ }^{4}$; Wilson Rogerio Boscolo ${ }^{4}$; Aldi Feiden ${ }^{4}$
}

\section{Resumo}

\begin{abstract}
O presente estudo teve como objetivo encontrar a melhor dose de eugenol para a anestesia do jundiá (Rhamdia voulezi) em diferentes classes de peso. Foram utilizados 240 jundiás distribuídos em delineamento experimental em blocos em esquema fatorial $(5 \times 4)$ totalizando 20 tratamentos, ou seja, cinco diferentes classes de peso: 32,$5 ; 75 ; 150 ; 300$ e $450 \mathrm{~g}$ e quatro concentrações de eugenol $\left(50,75,100\right.$ e $\left.125 \mathrm{mg} . \mathrm{l}^{-1}\right)$. Para cada tratamento foram utilizados 12 peixes escolhidos aleatoriamente $(\mathrm{n}=12)$ e expostos individualmente para cada concentração. Após o procedimento de anestesia os peixes foram transferidos para tanques-rede com $0,7 \mathrm{~m}^{3}$, onde receberam alimentação e ficaram em observação durante 96 horas para o monitoramento da mortalidade. O eugenol foi eficiente para a anestesia em jundiás nas diferentes concentrações analisadas e nos distintos pesos, e após 96 horas de acompanhamento à recuperação anestésica, não foram verificadas mortalidades dos animais. Nas condições deste experimento a melhor concentração de eugenol para indução e recuperação anestésica em jundiás com peso variando de 32,5 a $450 \mathrm{~g}$ é de $50 \mathrm{mg} \cdot \mathrm{l}^{-1}$.
\end{abstract}

Palavras-chave: Aquicultura, óleo de cravo, manejo de peixe

\begin{abstract}
The study aimed to find the better concentration of eugenol for anesthesia of silver catfish (Rhamdia voulezi) with different weights. Were used 240 catfish distributed in randomized blocks in factorial scheme (5x4) total 20 treatments, in others words, five different weights: 32,5; 75; 150; 300 e 450g and four eugenol concentrations: 50, 75, 100 e $125 \mathrm{mg}^{-1} \mathrm{l}^{-1}$. For each treatment were used 12 fish randomly chosen and exposed individually for each concentration. After of anesthesia the fish were transferred for net-cage with $0,7 \mathrm{~m}^{3}$, being fed and observed by 96 hours for monitoring of mortality. The eugenol was efficient for anesthesia in silver catfish all concentrations and weights and after of 96 hours no mortality have been verified. At these experimental conditions the best concentration of eugenol for anesthetic inducing and recuperation of silver catfish with weight varying from 32,5 and $450 \mathrm{~g}$ is $50 \mathrm{mg} . \mathrm{l}^{-1}$.
\end{abstract}

Key words: Aquaculture, clove oil, fish management

\footnotetext{
${ }^{1}$ Discente do Programa de Pós-Graduação em Aqüicultura, Universidade Estadual Paulista, Centro de Aquicultura, CAUNESP, Jaboticabal, SP. E-mail: odairdiemer@hotmail.com

${ }^{2}$ Discente do Programa de Pós-Graduação em Zootecnia, Universidade Estadual de Maringá, UEM, Maringá, PR. E-mail: dacley_ pesca@hotmail.com

${ }^{3}$ Discente do Programa de Pós-Graduação em Aqüicultura, Universidade Estadual Paulista, Centro de Aqüicultura, CAUNESP, Jaboticabal, SP. E-mail: bitanca@hotmail.com

${ }^{4}$ Profs. da Universidade Estadual do Oeste do Paraná, UNIOESTE, Toledo, PR.E-mail: altevir.signor@gmail.com; wilsonboscolo@ hotmail.com.br; aldifeiden@gmail.com

* Autor para correspondência
} 


\section{Introdução}

O jundiá é um bagre de água doce, onívoro que ocorre desde a região central da Argentina até o sul do México, porém, a espécie (Rhamdia voulezi) é endêmica do rio Iguaçu (REIS et al., 2011). Esta espécie tem despertado amplo interesse para a piscicultura da região Sul do Brasil por seu crescimento acelerado, inclusive nos meses mais frios, sua excelente eficiência alimentar e sua resistência ao manejo, além de carne saborosa, sem espinhos intramusculares. Existe boa oferta desta espécie na região Sul, onde tem sido cultivada com razoável sucesso (CANTON et al., 2007).

A piscicultura é uma atividade que esta em grande expansão no Brasil. No entanto, há grandes prejuízos devido à mortalidade decorrente do manejo inadequado (KUBITZA, 1998). Algumas práticas realizadas na aquicultura como biometria, reprodução induzida e transporte, frequentemente expõem peixes a várias fontes de estresse, consequentemente afetando o desempenho (VIDAL et al., 2008). O estresse provoca diminuição da alimentação, aumenta a susceptibilidade dos animais a patógenos e a doenças infecciosas (INOUE; SANTOS-NETO; MORAES, 2003).

Para evitar o estresse, anestésicos estão sendo utilizados durante o manejo. Contudo, há poucas informações sobre as concentrações que devem ser utilizadas para as diferentes espécies de peixes nativos criados no Brasil. Como cada anestésico exige uma concentração diferente para induzir ao estágio anestésico desejado, é necessário testar várias concentrações antes do tratamento definitivo para não ocorrer mortalidade dos animais expostos ao fármaco (ROUBACH; GOMES, 2001).

De acordo com os critérios de eficiência recomendados por Ross e Ross (2008), um anestésico deve ser eficaz em baixas concentrações e apresentar toxicidade em doses muito superiores às efetivas. Sua escolha deve ser baseada nos critérios de disponibilidade, custo, facilidade de utilização e segurança para o usuário e principalmente para o ambiente, características encontradas no eugenol (IVERSEN et al., 2003).

O eugenol, substância ativa do óleo de cravo, têm demonstrado ser eficiente e seguro como anestésico para peixes (TAYLOR; ROBERTS, 1999). Além disso, apresenta boa velocidade de ação, pois induz rapidamente à anestesia profunda (HAJEK; KLYSZEJKO; DZIAMAN, 2006). O presente trabalho teve como objetivo encontrar a melhor dose resposta do eugenol para a indução e recuperação do jundiá (Rhamdia voulezi) em diferentes classes de peso.

\section{Materiais e Métodos}

O trabalho foi realizado no dia 1 a 24 de Agosto 2010, desenvolvido na área aquícola do reservatório da Usina Hidrelétrica Governador José Richa (Salto Caxias) - rio Iguaçu, localizado no município de Boa Vista da Aparecida - PR e contou com o apoio do Grupo de Estudos em Manejo na Aquicultura (GEMAq) da Universidade Estadual do Oeste do Paraná (Unioeste).

Foram utilizados 240 jundiás distribuídos em delineamento experimental em blocos em esquema fatorial (5 x 4) totalizando 20 tratamentos, ou seja, cinco diferentes classes de peso: 32,$5 ; 75 ; 150$; 300 e $450 \mathrm{~g}$ e quatro concentrações de eugenol (50, 75,100 e $\left.125 \mathrm{mg} \cdot \mathrm{L}^{-1}\right)$. Para cada tratamento foram utilizados 12 peixes escolhidos aleatoriamente $(\mathrm{n}=12)$ e expostos individualmente para cada concentração.

Quinze dias antes do período experimental foi realizado uma classificação dos peixes, para isto, utilizaram-se cincos tanques-rede com volume útil de $5 \mathrm{~m}^{3}$, cada tanque-rede foi composto por 48 peixes de uma classe de peso.

Após os quinze dias de aclimatação, foram iniciados os procedimentos de anestesia, no qual, teve duração de cinco dias, para cada dia experimental foi submetido uma classe de peso e as quatro concentrações de eugenol. 
No dia do procedimento de anestesia foram capturados todos os peixes de uma classe de peso, sendo retirado do tanque-rede e transportado para uma caixa d'água circular de 1000L, mas contendo apenas 250L de água com renovação de água constante, sendo a água retirada do próprio reservatório onde estavam os peixes através de bombeamento.
Para a anestesia foi capturado aleatoriamente um peixe de cada vez e, posteriormente foi conduzido para uma caixa branca com volume útil de $30 \mathrm{~L}$, mas contendo apenas $10 \mathrm{~L}$ de água com a respectiva dose de anestésico. Os tempos necessários para o aparecimento dos padrões comportamentais avaliados foram monitorados por meio de cronômetro digital como descrito na Tabela 1.

Tabela 1. Características comportamentais dos peixes de acordo com os diferentes estágios de anestesia.

\begin{tabular}{|c|c|}
\hline Estágio & Característica de comportamento \\
\hline I & Movimento opercular visivelmente lento ou errático \\
\hline II & Perda parcial de equilíbrio e dificuldade de manter posição normal de nado, quando parado \\
\hline III & $\begin{array}{l}\text { Perda total de equilíbrio e incapacidade de recuperar a posição vertical de nado ("barriga para } \\
\text { cima") }\end{array}$ \\
\hline IV & Ausência de reação a qualquer estímulo \\
\hline Recuperado & Recuperação da posição normal de nado e da capacidade de nadar \\
\hline
\end{tabular}

Fonte: Woody, Nelson e Ramstad (2002).

A ausência de reação a qualquer estimulo foi verificada pelo toque na lateral dos peixes com um bastão de vidro. Quando os animais atingiam o estágio IV (tabela 1) foram retirados do aquário de indução, secos, medidos e pesados a fim de simular o processo de manejo e depois transferidos para o aquário de recuperação.

A recuperação anestésica dos peixes foi realizada individualmente, em uma caixa branca com volume útil de 30L, mas contendo apenas 20L de água, sem anestésico, onde os peixes foram acondicionados e o tempo de recuperação aferido.

Após o restabelecimento dos indivíduos no aquário de recuperação, os mesmos foram transferidos para tanques-rede com $0,7 \mathrm{~m}^{3}$, onde receberam alimentação e ficaram em observação durante 96 horas após indução anestésica para o monitoramento da mortalidade (VIDAL et al., 2006).

Os parâmetros físico-químicos da água do reservatório, temperatura, oxigênio dissolvido, $\mathrm{pH}$ e condutividade elétrica foram monitorados diariamente por aparelhos eletrônicos.
Para a análise estatística do efeito da concentração do eugenol sobre os peixes foi comparada a média dos valores encontrados por meio de análise de variância (ANOVA) e teste de média com nível de significância de 5\%, através do programa estatístico SISVAR versão 5.3 (FERREIRA, 2010).

\section{Resultados e Discussão}

Os parâmetros físicos e químicos da água do reservatório, temperatura $23 \pm 1,25{ }^{\circ} \mathrm{C}$, oxigênio dissolvido $6,93 \pm 0,67 \mathrm{mg} \cdot \mathrm{L}^{-1}, \mathrm{pH}$ 7,44 $\pm 0,27$ e condutividade elétrica $28,79 \pm 7,59 \mu \mathrm{S} . \mathrm{cm}^{-1}$ permaneceram dentro da faixa de condição ideal para criação de peixes tropicais (ARANA, 2004).

O eugenol foi eficiente para a anestesia em jundiás nas diferentes concentrações analisadas e nos distintos pesos, ou seja, em todas as concentrações avaliadas os peixes atingiram completamente o estágio IV, que é caracterizada pela ausência de reação a qualquer estímulo (Tabela 2). Ainda, depois de 96 horas de acompanhamento após a recuperação anestésica não foram verificadas mortalidades dos peixes. 
Tabela 2. Valores médios do tempo de indução e recuperação para cada classe de peso com suas doses anestésicas.

\begin{tabular}{|c|c|c|c|c|c|c|}
\hline & \multirow{2}{*}{$\begin{array}{c}\text { Classes de } \\
\text { peso (g) }\end{array}$} & \multicolumn{4}{|c|}{ Doses de eugenol $\left(\mathrm{mg.l}^{-1}\right)$} & \multirow[b]{2}{*}{ CV (\%) } \\
\hline & & 50 & 75 & 100 & 125 & \\
\hline \multirow{5}{*}{$\begin{array}{c}\text { Indução } \\
\text { (segundos) }\end{array}$} & 32,5 & $230,00^{\mathrm{aA}}$ & $209,17^{\mathrm{aA}}$ & $130,08^{\mathrm{abA}}$ & $115,83^{\mathrm{bAB}}$ & 26,03 \\
\hline & 75,0 & $177,25^{\mathrm{abA}}$ & $192,75^{\mathrm{abA}}$ & $123,25^{\mathrm{bcA}}$ & $93,67^{\mathrm{cA}}$ & 21,10 \\
\hline & 150,0 & $292,17^{\mathrm{aA}}$ & $211,00^{\mathrm{abA}}$ & $131,42^{\mathrm{abA}}$ & $102,5^{\mathrm{bA}}$ & 29,01 \\
\hline & 300,0 & $185,83^{\mathrm{aA}}$ & $179,08^{\mathrm{abA}}$ & $133,33^{\mathrm{abA}}$ & $112,75^{\mathrm{bA}}$ & 21,86 \\
\hline & 450,0 & $153,08^{\mathrm{aA}}$ & $164,67^{\mathrm{aA}}$ & $148,17^{\mathrm{aA}}$ & $167,25^{\mathrm{aB}}$ & 25,92 \\
\hline \multicolumn{2}{|c|}{ CV $(\%)$} & 34,05 & 22,47 & 25,88 & 20,78 & \\
\hline \multirow{5}{*}{$\begin{array}{c}\text { Recuperação } \\
\text { (segundos) }\end{array}$} & 32,5 & $74,75^{\mathrm{cA}}$ & $189,58^{\mathrm{abAB}}$ & $251,00^{\mathrm{aAB}}$ & $170,25^{\mathrm{bA}}$ & 25,13 \\
\hline & 75,0 & $75,08^{\mathrm{cA}}$ & $105,08^{\mathrm{bcA}}$ & $154,33^{\mathrm{abA}}$ & $215,33^{\mathrm{aA}}$ & 14,20 \\
\hline & 150,0 & $103,17^{\mathrm{bAB}}$ & $139,00^{\mathrm{abA}}$ & $151,83^{\mathrm{abA}}$ & $211,17^{\mathrm{aA}}$ & 23,67 \\
\hline & 300,0 & $109,67^{\mathrm{cB}}$ & $276,50^{\mathrm{bcB}}$ & $255,75^{\mathrm{abAB}}$ & $409,08^{\mathrm{aB}}$ & 25,21 \\
\hline & 450,0 & $100,00^{\mathrm{cAB}}$ & $199,42^{\text {bcAB }}$ & $326,00^{\mathrm{abB}}$ & $431,33^{\mathrm{aB}}$ & 42,15 \\
\hline \multicolumn{2}{|c|}{ CV (\%) } & 14,16 & 25,69 & 39,44 & 28,25 & \\
\hline
\end{tabular}

Médias na mesma coluna seguidos da mesma letra maiúscula e médias na mesma linha seguidos da mesma letra minúscula não diferem estatisticamente $(\mathrm{P}>0,05)$ pelo teste de Tukey.

Fonte: Elaboração dos autores.

As distintas concentrações de eugenol não foram significativamente diferentes $(\mathrm{P}>0,05)$ para induzir o animal à indução anestésica, com exceção da concentração com $125 \mathrm{mg} . \mathrm{l}^{1-1}$, que diferiu entre as classes de peso $(\mathrm{P}<0,05)$. Para a recuperação dos animais, a concentração do anestésico apresentou efeito significativo em todas as classes de peso $(\mathrm{P}<0,05)$.

Após o primeiro contato dos peixes com o anestésico, eles apresentavam movimentos lentos e desequilíbrio natatório até o momento em que não apresentaram nenhum movimento e perda total de reação aos estímulos, confirmados com o toque do bastão de vidro na lateral do peixe.

O presente estudo evidencia que conforme se aumenta a dose do anestésico, há queda no tempo de indução que tende a ir se estabilizando. Neste mesmo sentido, Walsh e Pease (2002), avaliando a eficácia do eugenol como anestésicos em enguias (Anguilla reinhardtii), também verificaram que o aumento na concentração do óleo de cravo durante a anestesia reduziu o tempo de indução dos animais ao anestésico. Contudo, todas as concentrações analisadas foram eficazes para a anestesia profunda, e, ainda as doses avaliadas estão dentro do intervalo proposto para algumas espécies como truta arcoíris (Oncorhynchus mykiss), 40-120 mg.1 ${ }^{-1}$, e carpa comum (Cyprinus carpio), 40-100 mg. $\mathrm{l}^{-1}$ (COYLE; DURBOROW; TIDWELL, 2005).

Os resultados demonstram que os peixes das menores classes de peso apresentam um período de indução bastante próximo aos peixes das maiores classes. Concordando com Vidal et al. (2007), que estudaram a influência do peso em juvenis de matrinxã (Brycon cephalus) apresentando pesos mínimos e máximos entre: 3,08 à 38,45 g e juvenis de tambaqui (Colossoma macropomum), apresentando pesos mínimos e máximos entre: 1,32 à 33,03 g, utilizando uma dose de $50 \mathrm{mg} \cdot \mathrm{l}^{-1} \mathrm{de}$ eugenol, concluíram que não houve influência do peso corporal de juvenis de matrinxã e de tambaqui sobre o tempo de indução.

O tempo de recuperação dos peixes com maior peso apresenta um tempo significativamente maior de recuperação, principalmente quando se eleva as concentrações de anestésicos. A mesma situação foi relatada por Vidal et al. (2006) quando estudaram a utilização do eugenol como anestésico em juvenis 
de pintado (Pseudoplatystoma corruscans), quando anestesiados com $50 \mathrm{mg} .1^{-1}$ de eugenol retornaram à posição normal mais rapidamente que os tratamentos com 75 e 100 mg..$^{-1}$ de eugenol.

Quando se observa o tempo de recuperação dos peixes em função da concentração do anestésico na água, nota-se que com o aumento da concentração do anestésico ocorre uma elevação do tempo de recuperação. O tempo ideal para a recuperação dos peixes deve ser inferior a 5 minutos (ROUBACH; GOMES, 2001). Contudo, as doses de 100 e 125 mg. $1^{-1}$ para as classes de 300 e 450 g estão acima desse intervalo de tempo indicando que ficam fora da margem de segurança da concentração ideal, portanto é aconselhável empregar doses menores.

Peixes com menores pesos apresentam um tempo de recuperação significativamente menor, isto deve estar relacionado com o metabolismo, pois peixes menores apresentam um metabolismo mais acelerado em comparação aos peixes maiores, o que faz com que estes se recuperam mais rapidamente que os peixes maiores (BARBOSA; MORAES; INOUE, 2007).

A concentração de eugenol mais efetiva neste experimento para todas as classes de pesos avaliadas, levando em consideração o tempo de indução e de recuperação foi a dose de $50 \mathrm{mg} .1^{-1}$, sendo resultado semelhante ao reportado por Cunha (2007), estudando a anestesia em jundiás (Rhamdia quelen) expostos a substâncias isoladas de plantas. O autor aconselha a utilização do óleo de cravo na faixa de 20 a $50 \mathrm{mg} .1^{-1}$ para indução à anestesia em jundiás, sendo que a concentração de $50 \mathrm{mg} \cdot \mathrm{l}^{-1}$ induz rapidamente a anestesia sem causar mortalidade durante a indução ou recuperação anestésica.

A anestesia proporcionada pelo eugenol facilitou o manejo dos peixes, além de não apresentar efeitos adversos aparente à saúde dos animais. Desta maneira, seu uso pode ser realizado na anestesia de jundiás.

\section{Conclusões}

Nas condições deste experimento a melhor concentração de eugenol para indução e recuperação anestésica em jundiás com peso variando de 32,5 a $450 \mathrm{~g}$ é de $50 \mathrm{mg} \cdot \mathrm{l}^{-1}$.

\section{Referências}

ARANA, L. V. Fundamentos da aqüicultura. Florianópolis: UFSC, 2004. 349 p.

BARbosA, L. G.; MORAES, G.; INOUE, L. A. K. Respostas metabólicas do matrinxã submetidos a banho anestésico de eugenol. Acta Scientiarum Biological Science, Maringá, v. 29, n. 3, p. 255-260, 2007.

CANTON, R.; WEINGARTNER, M.; FRACALOSSI, D. M.; ZANIBONI FILHO, E. Z. Influência da freqüência alimentar no desempenho de juvenis de jundiá. Revista Brasileira de Zootecnia, Viçosa, MG, v. 36, n. 4, p. 749753, 2007.

COYLE, S. D.; DURBOROW, R. M.; TIDWELL, J. H. Anesthetics in aquaculture. Disponível em: $<$ www.aces. edu/dept/fisheries/aquaculture/documents/58641543900fs.pdf. $>$. Acesso em: 28 abr. 2011.

CUNHA, M. A. Anestesia em jundiás (Rhamdia quelen) expostos a substâncias isoladas de plantas. 2007. Dissertação (Mestrado em Zootecnia) - Universidade Federal de Santa Maria, Santa Maria.

FERREIRA, D. F. Sisvar: versão 5.3. Lavras: UFLA, 2010.

HAJEK, G. J.; KLYSZEJKO, B.; DZIAMAN, R. The anaesthetic effect of clove oil on common carp, Cyprinus carpio L. Acta Ichthyologica et Piscatoria, Szczecin, v. 36, n. 2, p. 93-97, 2006.

INOUE, L. A. K. A.; SANTOS-NETO, C.; MORAES, G. Clove oil as anaesthetic for juveniles of matrinxã Brycon cephalus (Gunther, 1869). Ciência Rural, Santa Maria, v. 33, n. 5, p. 943-947, 2003.

IVERSEN, M.; FINSTAD, B.; MCKINLEY, S. R.; ELIASSEN, A. R. The efficacy of metomidate, clove oil, Aqui-Sk and BenzoakR as anaesthetics in atlantic salmon (Salmo salar L.) smolts, and their potential stressreducing capacity. Aquaculture, Amsterdãn, v. 221, n. 1-4, p. 549-566, 2003.

KUBITZA, F. Qualidade da água na produção de peixes. Campo Grande: [s.n.], 1998. 60 p. 
REIS, E. S.; FEIDEN, A.; SIGNOR, A.; ZAMINHAN, M.; FINKLER, J. K.; BOSCOLO, W. R. Suplementação de vitamina c na dieta para larvas de jundiá Rhamdia voulezi. Ciência Animal Brasileira, Goiânia, v. 12, n. 1, p. 83-89, jan./mar. 2011.

ROUBACH, R.; GOMES, L. C. O uso de anestésicos durante o manejo de peixes. Panorama da Aquicultura. Rio de Janeiro, v. 11, n. 66, p. 37-40, 2001.

TAYLOR, P. W.; ROBERT, S. D. Clove oil: an alternative anaesthetic for aquaculture. North American Journal of Aquculture, Bethesda, v. 61, n. 2, p. 150-155, 1999.

VIDAL, L. V. O.; ALBINATI, R. C. B.; ALBINATI, A. C. L.; LIRA, A. D.; ALMEIDA, T. R.; SANTOS, G. B. Eugenol como anestésico para a tilápia-do-nilo. Pesquisa Agropecuária Brasileira, Brasília, v. 43, n. 8, p. 10691074, 2008.
VIDAL, L. V. O.; ALBINATI, R. C. B.; SANTOS NETO, E. B.; DEUS, B. T.; ALBINATI, A. C. L. Influência do peso de juvenis de matrinxã (Brycon cephalus) e tambaqui (Colossoma macropomum) à ação anestésica do eugenol. Revista Brasileira de Saúde e Produção Animal, Salvador, v. 8, n. 3, p. 212-216, jul./set. 2007.

VIDAL, L. V. O.; ALBINATI, R. C. B.; LUSCHER,A. C.; MECÊDO, G. R. Utilização do eugenol como anestésico para o manejo de juvenis de Pintado (Pseudoplatystoma coruscans). Acta Scientiarum Biological Sciences, Maringá, v. 28, n. 3, p. 275-279, 2006.

WALSH, C. T.; PEASE, B. C. The use of clove oil as an anaesthetic for the longfinned eel, Anguilla reinhardtii. Aquaculture Research, Oxford, v. 33, n. 8, p. 627-635, 2002.

WOODY, C. A.; NELSON, J.; RAMSTAD, K. Clove oil as an anaesthetic for adult sockeye salmon: field trials. Journal of Fish Biology, Liverpool, v. 60, n. 2, p. 340347, 2002. 